\title{
Lungenfunktionsnormwerte neu berechnet
}

\author{
Die Spirometrie ist die wichtigste \\ und global gebräuchlichste Mess- \\ methode zur Quantifizierung der \\ Lungenfunktion. Jetzt wurden neue, \\ an Gesunden erfasste Normwerte \\ von der European Respiratory Society \\ (ERS) publiziert.
}

— Die bisher für die Lungenfunktionsmessung verwendeten Normwerte stammen von der European Community for Steel and Coal (ECSC 1993). Allerdings ergaben sich mit diesen Normwertetabellen einige Probleme. So sind kleine Kinder und Jugendliche oder Personen jenseits des 80. Lebensjahres nur in geringen Zahlen erfasst worden. Die 2005 von der American Thoracic Society (ATS) und von der ERS publizierten Empfehlung zur Lungenfunktionsprüfung basierten auf Normwertemessungen an Weißen (Kaukasiern), AfroAmerikanern und Latinoamerikanern.

Seit 2006 wurden darüber hinaus weltweit Daten an nichtrauchenden
Gesunden gesammelt. Die beitragenden Zentren mussten bestimmten Qualitätsansprüchen gerecht werden und übermittelten ihre Daten zu der extra für diesen Zweck gegründeten „Global Lung Function Initiative" (GLI), die 2010 ERS Task Force-Status erhielt. 72 Zentren in 33 Ländern übermittelten Lungenfunktionsdaten von 97759 Nichtrauchern im Alter von 3-95 Jahren. Davon waren 55,3\% Frauen.

Die forcierte Ein-Sekundenkapazität $\left(\mathrm{FEV}_{1}\right)$ und die forcierte Vitalkapazität (FVC) differierten zwischen den verschiedenen ethnischen Gruppen. Das Verhältnis zueinander $\left(\mathrm{FEV}_{1} / \mathrm{FVC}\right)$ blieb über alle Gruppen und Länder mehr oder weniger ähnlich. Die Berechnungen der Normwertetabellen enthalten jetzt auch alterskorrigierte, sog. ,lower limit of normal"- Werte für alle ethnischen Gruppen. Folgende Einflussfaktoren bestimmen den Normwert: Alter, Größe, Geschlecht und ethnische Gruppe.

\section{Hilft Avocado-Sojaöl bei Arthrose?}

\begin{abstract}
Avocado-Sojaöl als Nahrungsergänzungsmittel scheint nach einer neuen Studie die Progression einer Arthrose aufzuhalten.
\end{abstract}

- Pariser Rheumatologen randomisierten 399 Patienten mit Arthrose im Hüftgelenk in zwei Gruppen. Sie erhielten drei Jahre lang entweder Avocado-Sojaöl (300 mg/Tag) oder Placebo. Hauptzielparameter der Studie war die radiologisch bestimmte Weite des betroffenen Gelenkspalts. Dieser hatte am Ende der Therapiephase in der Experimentalgruppe im Vergleich mit der Kontrollgruppe um 20\% weniger abgenommen. Bei den klinischen Beschwerden der Patienten zeigten sich allerdings keine Gruppenunterschiede. Die Verträglichkeit des Verums wurde als hervorragend eingestuft.

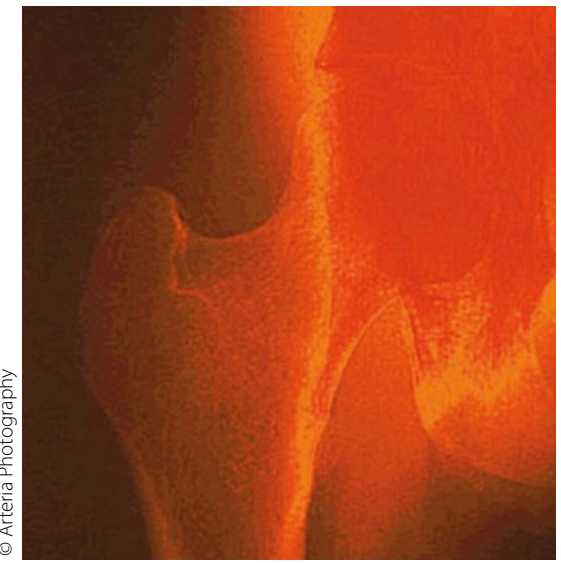

Verengter Gelenkspalt bei Hüftarthrose.

\footnotetext{
- E. Maheu et al.

Randomised, controlled trial of avocadosoybean unsaponifiable (Piascledine) effect on structure modification in hip osteoarthritis: the ERADIAS study. Ann Rheum Dis. 2013 Jan 23. [Epub ahead of print].
}

\section{Kommentar}

Die Erhebung von Normwertedaten für die Spirometrie war schon lange überfällig, da die Limitationen der ECSC-Daten bekannt waren. Jedes Spirometrie-Gerät und jeder Bodyplethysmograph vergleicht bis heute die gemessenen Werte mit den ECSC-Daten, aus denen sich dann die prozentualen $A b$ weichungen errechnen. Diese waren, wie diese Studie eindrucksvoll beweist, fehlerhaft.

A. GILLISSEN *
- P. H. Quanjer et al.

Multi-ethnic reference values for spirometry for the 3-95-yr age range: the global lung function 2012 equations. Eur Respir J 2012 40:1324-1343; published ahead of print 2012, doi:10.1183/09031936.00080312

\section{Kommentar}

Die Fachwelt war sich bislang uneins bezüglich der Wirksamkeit von Avocado-Sojaöl. Einige Studien lieferten positive Ergebnisse, während andere diese nicht bestätigen konnten. Die vorliegende, sehr gut gemachte Untersuchung könnte diesen Widerspruch vielleicht klären. Es scheint, als würde dieses Mittel das Fortschreiten der Arthrose im Gelenk verlangsamen, ohne jedoch die Symptomatik zu beeinflussen. Vielleicht ist die Behandlungsdauer von drei Jahren zu kurz, um auch hier einen Effekt zu sehen. Wie dem auch sei, es gibt herzlich wenig therapeutische Optionen, die Arthroseentwickung zu verlangsamen, und wir sollen daher das offenbar völlig nebenwirkungsfreie AvocadoSojaöl ernsthaft in Betracht ziehen.

E. ERNST : 